\title{
Exact solutions for a mean-field Abelian sandpile*
}

\author{
Steven A. Janowskył \\ Department of Mathematics \\ Rutgers University \\ New Brunswick, NJ 08903
}

\author{
Claude A. Laberge \\ Department of Physics \\ Rutgers University \\ Piscataway, NJ 08855-0849
}

June 1993

\begin{abstract}
We introduce a model for a sandpile, with $N$ sites, critical height $N$ and each site connected to every other site. It is thus a mean-field model in the spin-glass sense. We find an exact solution for the steady state probability distribution of avalanche sizes, and discuss its asymptotics for large $N$.
\end{abstract}

The publication of a series of articles by P. Bak and collaborators [1], 2] generated a growing interest in the study of certain class of cellular automata models now commonly known as "sandpiles" because of a crude analogy between the dynamical rules and the way sand topples when building a sandpile. The basic motive was not to model the way real sandpiles behave, although it did broaden the audience for granular flow (see e.g. [3, 4, 5]), but to study a phenomenon they coined "self-organized criticality" in which a system reaches a critical state, i.e. a state with no intrinsic length or time scales, without the tuning of an external parameter. This generated a big influx of theoretical, computational and even experimental studies of selforganized criticality and of sandpiles in general. Unfortunately, even though the number of models (i.e. sets of dynamical rules) exhibiting self-organized criticality or falling into the more general category of sandpiles has increased dramatically, very few exact results are known at the present (see e.g. [6, 7, 8, 9, 10]). In this letter we present a model in which many quantities can be calculated exactly in a rigorous way.

\footnotetext{
*Supported in part by NSF Grant DMR89-18903

${ }^{\dagger}$ Supported in part by NSF Mathematical Sciences Postdoctoral Research Fellowship DMS 90-07206

${ }^{\ddagger}$ Address after August 1993: Department of Mathematics, University of Texas, Austin, TX 78712
} 
A sandpile model is basically a set of dynamical rules describing the way that grains of sand are added to a system, the conditions under which those grains can be redistributed inside the system, and the way they are removed from the system. Here we consider a system of $N$ sites and define $h(i)$ as the (integer) height of the column of sand at site $i, i \in\{1 \ldots N\}$. We drop a grain of sand on a site $i$ chosen at random, thereby increasing its height by one: $h(i) \rightarrow h(i)+1$. If this new height exceeds the maximum stable value $h_{c}$ then that column topples and gives 1 grain of sand to each of the $N-1$ other sites while one grains drops out of the system. (We take $h_{c} \geq N$ so that $h(i) \geq 0$; in fact we are primarily interested in $h_{c}=N$.) We then examine the system to see if any site has a column exceeding $h_{c}$ in which case we topple that column also. We keep toppling until all the sites are stable (this characterizes an avalanche). We then repeat the procedure of adding a grain at a randomly chosen site.

This model falls into the category of abelian sandpiles since we always obtain the same stable configuration from an unstable one irrespective of the order in which we executed the topplings. Dhar [1] used this fact to obtain several properties of those models which we use extensively in our analysis. Furthermore, since all sites are connected to all other sites, we can say that we have a mean field theory of abelian sandpiles. It is this combination that permits the model to be solved. It should be pointed out that our model is quite different from previous attempts to study mean-field sandpiles [12, 13].

It is shown in [11] that not all configurations are allowed in the asymptotic regime, but that the allowed (recurrent) configurations all have equal weight. In general, the number of recurrent configuration is given by the determinant of the toppling matrix $\Delta$ which is an $N \times N$ matrix where $\Delta_{i i}=h_{c}$ and $\Delta_{i j}=-1$ for each site $j$ connected to $i$, so that row $i$ of $\Delta$ represents the amount of sand lost by every site when site $i$ topples. Thus in the model considered here this matrix takes the form

$$
\Delta_{i j}=-1+\left(h_{c}+1\right) \delta_{i j}
$$

where $\delta_{i j}$ is the Kronecker delta.

Because of the highly symmetric nature of $\Delta$ in our model it is straightforward to calculate the determinant and determine that the number of recurrent configurations is

$$
Z\left(N, h_{c}\right)=\operatorname{det} \Delta=\left(h_{c}+1-N\right)\left(h_{c}+1\right)^{N-1}
$$


For the case $h_{c}=N$ we have $Z(N, N)=(N+1)^{N-1}$. Incidentally this is the number of spanning trees on a fully connected graph with $N+1$ sites. Such a result was expected from the general observation that $\operatorname{det} \Delta$ is precisely the cofactor of the matrix tree (see e.g. [14]) for graphs defined on a superset of the sandpile lattice (there is an additional site, the ground, connected to every site). In fact there is a one-to-one correspondence between the recurrent configurations and the spanning trees, in the sense that a configuration is recurrent if and only if it passes the "burning algorithm" test described in [6, 8]. Actually this holds only for symmetric abelian sandpiles, i.e. models where $\Delta$ is symmetric; for the asymmetric case see [15].

Clearly the recurrent configurations fall into equivalent classes, where two configurations are equivalent if one is a permutation of the other. Because of the symmetry of the model we need only examine one member of each equivalence class; we find it convenient to restrict ourselves to the case $h(i) \leq h(i+1), i=1 \ldots N-1$, where the amount of sand increases as the site label increases.

Our analysis will focus on a particular subset of the recurrent configurations, namely the minimal configurations. A given configuration is minimal if there is no recurrent configuration that can be obtained by removing sand from the given configuration. Some brief computations show (one can use the so-called "burning algorithm" of [6]) that the configuration $h(i)=i$ is a minimal configuration. All minimal configurations fall into this equivalence class and thus there are exactly $N$ ! minimal configurations, the permutations of $h(i)=i$. If we now consider equivalence classes for general configurations, we see that the restriction that a recurrent configuration be greater than or equal to the minimal configuration yields

$$
h(i) \geq i, \quad h(i) \geq h(i-1)
$$

$($ set $h(0)=0)$.

Now consider how avalanches of various sizes come about in our model; we limit the discussion to the case $h_{c}=N$. Size here means either the number of grains that fall out of the system or the number of sites that topple; in the model considered here there is no ambiguity because they are equal. An avalanche begins when a grain of sand is dropped on a site of height $N$. It will continue if the second-highest site is also at height $N$. It will be at least of size three if the third-highest site is at height $N-1$, and it will be at least of size four if 
the fourth-highest site is at height $N-2$, etc. Therefore the size will be (using the equivalence class notation)

$$
N_{\text {aval }}=N-\min \{j: h(i)>i \text { for all } j \leq i<N\}
$$

This is the only non-zero size avalanche this configuration can produce, and it is determined simply by "how long" the configuration stays away from a minimal one.

If we have such a configuration, what is the probability of starting an avalanche? It is simply the fraction of sites at the maximum value, $|\{i: h(i)=N\}| / N$. Therefore, to determine the avalanche size probability distribution, we merely have to count the configurations with fixed $N_{\text {aval }}$ and with a fixed number of sites at height $N$. Luckily, the number of such configurations can be simply represented as partition functions for smaller systems with different critical heights - we obtain (for $1<k \leq N$ ):

$$
\begin{aligned}
& P(\text { avalanche of size } k)= \\
& Z(N, N)^{-1} Z(N-k, N-k) \sum_{j=2}^{k}\left(\begin{array}{c}
N \\
j, k-j, N-k
\end{array}\right) \frac{j}{N} Z(k-j, k-2) .
\end{aligned}
$$

A brief explanation of the source of the terms in (5) is in order. First of all, since site $N-k$ must be at height $N-k$ to get an avalanche of the right size, the remaining sites below $N-k$ yield a factor $Z(N-k, N-k)$. Next $j$ is the number of sites at height $N$. The sites between $N-k+1$ and $N-j$ must be greater than minimal yet less than $N$; this is equivalent to $Z(k-j, k-2)$. The combinatoric factor gives us the number of ways we can choose configurations with fixed equivalence classes for the subsystems. Finally we have the probability of toppling $j / N$ and the normalization $Z(N, N)$.

Simpler expressions hold for $k=0,1$ :

$$
\begin{gathered}
P(\text { avalanche of size } 1)=Z(N-1, N-1) / Z(N, N) \\
P(\text { no avalanche })=\sum_{j=1}^{N}\left(\begin{array}{c}
N \\
j
\end{array}\right) \frac{N-j}{N} Z(N-j, N-1) / Z(N, N) .
\end{gathered}
$$

The above sums (5 7) can be performed exactly. The result is

$$
P(\text { avalanche of size } k)=\frac{(N-k+1)^{N-k-1} k^{k-2}(N-1) !}{(N+1)^{N-1}(k-1) !(N-k) !}
$$

for $1 \leq k \leq N$, and

$$
P(\text { no avalanche })=\frac{N-1}{N+1} .
$$


The probability distribution (8) has the unusual feature that (for $k \neq 0$ ) it is symmetric about $k=(N+1) / 2$.

We are interested in the large $N$ behavior of this result. Taking $k>0$ fixed and $N \rightarrow \infty$, (8) yields

$$
P(\text { avalanche of size } k) \sim \frac{1}{N} \frac{k^{k-2} e^{-k}}{(k-1) !},
$$

and if $\mathrm{k}$ is also large $(1 \ll k \ll N)$ then this reduces to

$$
P(\text { avalanche of size } k) \sim \frac{k^{-3 / 2}}{\sqrt{2 \pi} N} .
$$

Thus we reproduce the exponent $-3 / 2$ previously derived on trees [7] and via numerical and nonrigorous arguments [16, 17].

Another quantity of interest is the single-site probability distribution for the heights, i.e. the probability that a given site contains $H$ grains of sand in the stationary state. First look at configurations where exactly $K$ sites have height $H$. (In the equivalence class notation these sites must be consecutive.) Remove these sites and consider the subsystem consisting of the remaining $N-K$ sites. The recurrent configurations of this subsystem are in one-toone correspondence with those of a system of size $N-K$ where the recurrent configurations are determined by, in addition to the usual occupancy restrictions (see (3)), the conditions $j \leq h(j) \leq N-1$ if $j \leq H-K$ and $j+K-1 \leq h(j) \leq N-1$ if $j>H-K$.

The direct evaluation of the number of allowed configurations satisfying the above criteria is possible but somewhat complicated; appropriately summing over $K$ would yield the desired probabilities. However, it turns out to be simpler instead to compare the number of allowed configurations with $K$ sites having $H$ grains with the number of allowed configurations with $K$ sites having $H-1$ grains, e.g. by subtracting the latter from the prior. Which configurations are left?

In both cases $j \leq h(j) \leq N-1$ for $j<H-K$ and $j+K-1 \leq h(j) \leq N-1$ if $j>H-K$. The only difference occurs at site $H-K$ which can be occupied by between $H-1$ and $N-1$ grains in the first case, and between $H-K$ and $N-1$ grains in the second. If $K=1$ these conditions are identical and no allowed configurations are left. For $K \geq 2$, however, site $H-K$ is now restricted so that $h(H-K) \leq H-2$. Since we need $h(j+1) \geq h(j)$ (for the configuration to be a recurrent one), this implies that the first $H-K$ sites can have a maximum of $H-2$ grains 
each and thus contribute a factor $Z(H-K, H-2)$ to the partition function, excluding (global) symmetry factors. On the other hand, (3) imposes no additional conditions on the last $N-H$ sites, and it is easily seen that they contribute a factor $Z(N-H, N-H)$. Defining $P(H)=$ the probability that a given site will have $H$ grains, putting in the appropriate symmetry factors and summing over the index $K$ we get

$P(H)-P(H-1)=Z(N, N)^{-1} Z(N-H, N-H) \sum_{K=2}^{H}\left(\begin{array}{c}N \\ K, H-K, N-H\end{array}\right) \frac{K}{N} Z(H-K, H-2)$,

which is exactly (5)! Since $P(0)=0$ we can collapse the telescoping sum and obtain

$$
P(\text { site has } H \text { grains })=\sum_{k=1}^{H} P(\text { avalanche of size } \mathrm{k}) .
$$

Because of the symmetry of the avalanche distribution, we have here the symmetry

$$
P(\text { site has } H \text { grains })+P(\text { site has } N-H \text { grains })=\frac{2}{N+1}
$$

for $0 \leq H \leq N$.

The asymptotics of (13) are relatively easy to compute using our previous results. For $N \gg 1$ we have

$$
P(\text { site has } H \text { grains }) \sim \frac{1}{N} \sum_{k=1}^{H} \frac{k^{k-2} e^{-k}}{(k-1) !}
$$

which can be used directly to compute the behavior for small $H$. For $N \gg H \gg 1$ we have

$$
P(\text { site has } H \text { grains }) \sim \frac{1}{N+1}\left(1-\sqrt{\frac{2}{\pi H}}\right) .
$$

We can also examine the total amount of sand in the system. The distribution of masses (total number of grains of sand) of recurrent configurations is most easily computed for mass $M$ near the maximum value $N^{2}$. For example, the number of recurrent configurations with mass $N^{2}-2$ is simply $N(N+1) / 2$. This is easily generalized to arbitrary mass (using inclusionexclusion arguments) and we find that the number of recurrent configurations with total mass $N^{2}-K, Z_{N^{2}-K}(N, N)$, is

$$
\begin{aligned}
& Z_{N^{2}-K}(N, N)=\left(\begin{array}{c}
N-1+K \\
N-1
\end{array}\right)+ \\
& \quad \sum_{l=1}^{l(N, K)}(-1)^{l}\left(\begin{array}{c}
N \\
l
\end{array}\right) \sum_{j=l N+l(l-1) / 2}^{K}\left(\begin{array}{c}
N-l-1+K-j \\
N-l-1
\end{array}\right)\left(\begin{array}{c}
j-l N-l(l+1) / 2-1 \\
l-1
\end{array}\right),
\end{aligned}
$$


where

$$
l(N, K)=\left\lceil N-(N(N-5)+2 K+17 / 4)^{1 / 2}-3 / 2\right\rceil
$$

simply indicates "how far" one must go in the inclusion-exclusion; $\lceil A\rceil$ is the least integer $\geq A$. Then the probability of a configuration having mass $M$ is $Z_{M}(N, N) /(N+1)^{N-1}$. Unfortunately we are only able to find the asymptotic behavior of (17) for the tails of the distribution, i.e. $M$ near $N(N+1) / 2$ or $M$ near $N^{2}$, which is not where most of the (recurrent) configurations reside. Expressions similar to that which were used to find the avalanche distribution, e.g. (5), are available:

$$
Z_{M}\left(N, h_{c}\right)=\sum_{j=1}^{N} Z_{M-j h_{c}}\left(N-j, h_{c}-1\right)\left(\begin{array}{c}
N \\
j
\end{array}\right),
$$

and one could consider a generating function approach to eliminate the restrictions on $j$ in the sum. Unfortunately the resulting expression will only converge in the regions equivalent to very large or very small mass, and thus provide no new information.

The reformulation of the $N$-dimensional model in terms of a one dimensional sandpilelike problem (with the required symmetry factor attached to each configuration) greatly facilitated the computations of "static" quantities like the avalanche size, single-site height, and total mass distributions. In order to study the "dynamical" quantities associated to the evolution of an avalanche, another reformulation in term of a one dimensional particle model proves to be useful; we will use it to calculate the distribution of avalanche durations. This reformulation also provides a much more efficient method for numerical study than a straightforward implementation of the sandpile process.

The duration of an avalanche is the number of sweeps that are required in order to reach a stable configuration once a grain of sand is dropped on the system. A sweep consists of two steps: we first go through all the sites and mark those with a number of grains exceeding the critical value and then topple all those sites simultaneously. The process is repeated until no site can topple. Note that this definition of duration is not the only one; others are possible because of the abelian nature of the model.

The particle model consists of $N$ particles moving on a one dimensional lattice with sites labeled from left to right by $k \in[1, N+1]$ (where $\mathrm{N}$ is the number of site in the original model). The correspondence between sandpile configurations and particle configurations is as follows: given a sandpile configuration $C_{0}$ place a particle at position k for every site in $C_{0}$ containing k 
grains. If $n_{k}$ is the resulting number of particles at site $k$, the stability condition (3) translates to $n_{k} \leq k$ and $n_{N}>1$; since the critical height is $N$ we also have $n_{N+1}=0$.

The boundary conditions are "almost" periodic as will be explained below; we also introduce a marker (barrier) between sites $N$ and $N+1$ that will play an important role in the dynamics.

The dynamics of the particle model is as follow: pick a particle at random and move it one site to the right (this is equivalent of dropping a grain of a sand on $C_{0}$ ). If the barrier was not crossed then we have a new stable configuration and can repeat the step. Thus, neglecting the boundary, our model is a zero-range process; however, the boundary plays a very important role.

If the chosen particle jumps through the barrier it means that one site in $C_{0}$ is unstable and will topple. In the particle model such a toppling will mean that all particles simultaneously jump one site to the right except for the one at site $N+1$ which should move back to site 1 . An equivalent formulation which we find more convenient is to move the barrier to the left by one unit and relabel the sites accordingly (i.e. the barrier defines the position of site $\mathrm{N}$ and $\mathrm{N}+1$ on the periodic lattice).

As we moved the barrier, a number of particles (say $p_{2}$ ) might have gone through it. In the sandpile model this means that the initial toppling caused $p_{2}$ more sites to become unstable. To duplicate their toppling the barrier should now be moved $p_{2}$ more units to the left. The process continues until no particle crosses the barrier as it jumps. The total number of barrier jumps required is the duration of the avalanche. The probability that an avalanche has duration $T$ is equivalent to the fraction of all particle configurations that will make the barrier jump $T$ times if a particle at site $N$ is picked. Of course $T=0$ corresponds to the no avalanche case so that the probability that the avalanche duration is zero is $(N-1) /(N+1)($ see $(9))$.

If there are $T$ barrier jumps, let $p_{1}, p_{2}, \ldots p_{T}$ be the number of particles crossing the boundary at each jump and let $P=p_{1}+p_{2}+\cdots+p_{T}$; note that $p_{i}>0$ for $i<T$ and $p_{T}=0$; we also have $p_{1}>1$ for $T>1$. Provided $T>0$ we have

$$
P(\text { duration } T)=Z(N, N)^{-1} \sum_{p_{1}, \ldots, p_{T} \text { restricted }} \frac{p_{1}}{N} W_{N}\left(1 ; p_{1}, p_{2}, \ldots, p_{T}\right)
$$

where $W_{N}\left(1 ; p_{1}, p_{2}, \ldots, p_{T}\right)$ is the number of configurations that produce the specified barrier jumps. We can compute this in an iterative fashion, in terms of $W_{M}\left(s ; p_{i}, p_{i+1}, \ldots, p_{T}\right)$, the 
number of (restricted) configurations on the subsystem consisting of sites $1 \ldots M$; $s$ is size of the current jump, and $p_{i}, p_{i+1}, \ldots, p_{T}$ are the remaining jump sizes. We have

$$
\begin{aligned}
W_{N}\left(1 ; p_{1}, p_{2}, \ldots, p_{T}\right)= & \left(\begin{array}{c}
N \\
p_{1}
\end{array}\right) w\left(p_{1}, 1\right) W_{N-1}\left(p_{1}-1 ; p_{2}, p_{3}, \ldots, p_{T}\right), \\
W_{N-1}\left(p_{1}-1 ; p_{2}, p_{3}, \ldots, p_{T}\right)= & \left(\begin{array}{c}
N-p_{1} \\
p_{2}
\end{array}\right) w\left(p_{2}, p_{1}-1\right) W_{N-p_{1}}\left(p_{2} ; p_{3}, p_{4}, \ldots, p_{T}\right), \\
& \text { and } \\
W_{M}\left(p_{i-1} ; p_{i}, p_{i+1}, \ldots, p_{T}\right)= & \left(\begin{array}{c}
M \\
p_{i}
\end{array}\right) w\left(p_{i}, p_{i-1}\right) W_{M-p_{i-1}}\left(p_{i} ; p_{i+1}, p_{i+2}, \ldots, p_{T}\right)
\end{aligned}
$$

for $2<i<T-1$. The weight $w(p, s)$ is easily computed since the criteria for allowed configurations are automatically satisfied within each block. Since each of the $p$ particles can independently be at any of the $s$ sites,

$$
w(p, s)=s^{p} .
$$

Finally, the last step of the iteration is simply

$$
W_{N-P+p_{T-1}}\left(p_{T-1} ; p_{T}\right)=\left(\begin{array}{c}
N-P+p_{T-1} \\
p_{T}
\end{array}\right) w\left(p_{T}, p_{T-1}\right) Z(N-P, N-P)=Z(N-P, N-P) .
$$

As an example, for $T=4$ this would lead to

$$
P(\text { duration } 4)=\sum_{p_{1}=2}^{N-2} \sum_{p_{2}=1}^{N-p_{1}-1} \sum_{p_{3}=1}^{N-p_{1}-p_{2}} \frac{Z(N-P, N-P)}{Z(N, N)} \frac{p_{1}}{N}\left(\begin{array}{c}
N \\
p_{1} p_{2} p_{3} p_{4}
\end{array}\right)\left(p_{1}-1\right)^{p_{2}} p_{2}^{p_{3}} .
$$

The general case can actually be written in a reasonably compact form. We can rearrange the terms and obtain the following (for $T>0$ ):

$$
P(\text { duration } T)=\frac{1}{(N+1)^{N-1}} \sum_{S=T}^{N} \frac{1}{N}\left(\begin{array}{c}
N \\
S
\end{array}\right)(N-S+1)^{N-S-1} \sum_{s_{1}+s_{2}+\cdots+s_{T}=S} \frac{S !}{s_{1} ! \ldots s_{T} !} s_{1}^{s_{2}} \ldots s_{T-1}^{s_{T}}
$$

where we have the restriction $s_{1}=1, s_{i}>0$. We identify $s_{i}$ with $p_{i-1}$ except that because of the need to initiate the avalanche $s_{1}=1$ and $s_{2}=p_{1}-1$. Of course $s_{i}$ is nothing more than the size of the $i^{\text {th }}$ jump.

\section{Acknowledgments:}

We would like to thank J. L. Lebowitz, S. Majumdar and E. R. Speer for their help with this work. 


\section{References}

[1] Bak P, Tang C and Wiesenfeld K 1987 Self-organized criticality:An explanation of $1 / f$ noise Phys. Rev. Lett. 59 381-384

[2] Bak P, Tang C and Wiesenfeld K 1988 Self-organized criticality Phys. Rev. A 38 364-374

[3] Nagel S R 1992 Instabilities in a sandpile Rev. Mod. Phys. 64 321-325

[4] Mehta A 1992 Real sandpiles: dilatancy, hysteresis and cooperative dynamics Physica A $186121-153$

[5] Puhl H 1992 On the modelling of real sand piles Physica A 182 295-319

[6] Majumdar S N and Dhar D 1991 Height correlations in the Abelian sandpile model $J$. Phys. A 24 L357-L362

[7] Dhar D and Majumdar S N 1990 Abelian sandpile model on the Bethe lattice J. Phys. A $234333-4350$

[8] Majumdar S N and Dhar D 1992 Equivalence of the Abelian Sandpile Model and the $q \rightarrow 0$ Limit of the Potts Model Physica A 185 129-145

[9] Dhar D and Ramaswamy R 1989 Exactly Solved Model of Self-Organized Critical Phenomena Phys. Rev. Lett. 63 1659-1662

[10] Lee S-C, Liang N Y and Tzeng W-J 1991 Exact Solution of a Deterministic Sandpile Model in One Dimension Phys. Rev. Lett. 67 1479-1481

[11] Dhar D 1990 Self-Organized Critical State of Sandpile Automaton Models Phys. Rev. Lett. $641613-1616$

[12] Tang C and Bak P 1988 Mean Field Theory of Self-Organized Critical Phenomena J. Stat. Phys. 51 797-802

[13] Gaveau B and Schulman L S 1991 Mean-field self-organized criticality J. Phys. A 24 L475-L480

[14] Harary F 1990 Graph Theory (Reading, MA: Addison-Wesley)

[15] Speer E R 1993 Asymmetric Abelian Sandpile Models J. Stat. Phys. 71 61-74

[16] Obukov S P 1988 in Random Fluctuations and Pattern Growth: Experiments and Models, ed. H E Stanley and N Ostrowsky, NATO ASI Series C 157 (Dordrecht:Kluwer)

[17] Kadanoff L P, Nagel S R, Wu L and Zhou S-M 1989 Scaling and universality in avalanches Phys. Rev. A 39 6524-6537 